\title{
Combined microvascular breast and lymphatic reconstruction with deep inferior epigastric perforator flap and gastroepiploic vascularized lymph node transfer for postmastectomy lymphedema patients
}

\author{
Pedro Ciudad ${ }^{1,2}$, Oscar J. Manrique ${ }^{3,4}$, Samyd S. Bustos ${ }^{3,4}$, Maria I. Vargas ${ }^{1}$, César Reynaga ${ }^{1}$, \\ Mouchammed Agko ${ }^{5}$, Tony C. T. Huang ${ }^{3}$, Eduardo Figueroa Benites $^{6}$, Horacio F. Mayer ${ }^{7}$, \\ Antonio J. Forte ${ }^{8}$
}

${ }^{1}$ Department of Plastic, Reconstructive and Burn Surgery, Arzobispo Loayza National Hospital, Lima, Peru; ${ }^{2}$ Department of Plastic and Reconstructive Surgery, Ciruesthetic Clinic, Lima, Peru; ${ }^{3}$ Division of Plastic and Reconstructive Surgery, ${ }^{4}$ Center for Regenerative Medicine, Mayo Clinic, Rochester, MN, USA; ${ }^{5}$ Section of Plastic Surgery, Medical College of Georgia, Augusta University, Augusta, GA, USA; ${ }^{6}$ Department of Oncology, Arzobispo Loayza National Hospital, Lima, Peru; ${ }^{7}$ Plastic Surgery Department, Hospital Italiano de Buenos Aires, University of Buenos Aires Medical School, Buenos Aires, Argentina; ${ }^{8}$ Division of Plastic and Reconstructive Surgery, Mayo Clinic, Jacksonville, FL, USA Contributions: (I) Conception and design: P Ciudad; (II) Administrative support: P Ciudad, OJ Manrique, SS Bustos, AJ Forte; (III) Provision of study materials or patients: P Ciudad, OJ Manrique; (IV) Collection and assembly of data: P Ciudad, OJ Manrique, SS Bustos, MI Vargas; (V) Data analysis and interpretation: P Ciudad, OJ Manrique, SS Bustos, TCT Huang; (VI) Manuscript writing: All authors; (VII) Final approval of manuscript: All authors.

Correspondence to: Pedro Ciudad, MD, PhD. Department of Plastic, Reconstructive and Burn Surgery, Arzobispo Loayza National Hospital, Lima, Peru. Email: pciudad@hotmail.com.

Background: The combination of microvascular breast reconstruction (MBR) and vascularized lymph node transfer (VLNT) in a single-stage procedure is a surgical option for women who desire breast reconstruction and postmastectomy lymphedema surgery. In this study, we present a series of patients who underwent simultaneous lymphatic and MBR with the gastroepiploic VLNT (GE-VLNT) and the deep inferior epigastric perforator (DIEP) flap respectively.

Methods: Between 2018 and 2019, all consecutive patients diagnosed with lymphedema stage IIb-III International Society of Lymphology who opted to pursue simultaneous MBR with DIEP flap and GEVLNT were included in this study. Patient demographics, comorbidities, prior radiation therapy, operative characteristics, surgical outcomes and complications were collected and analyzed.

Results: Six patients underwent simultaneous unilateral MBR with DIEP flap and GE-VLNT. The mean age was $48 \pm 10.5$ years and mean body mass index was $28.2 \pm 4.5 \mathrm{~kg} / \mathrm{m}^{2}$. The flap survival rate was $100 \%$. One patient required re-exploration due to venous congestion of the lymph node flap but was successfully salvaged. There was no donor site morbidity at the donor or recipient site for the DIEP flap were seen. The mean circumference reduction rate was $30.0 \% \pm 5.1 \%(\mathrm{P}<0.001)$. One patient stage III underwent additional liposuction at 12 months postoperative to improve final results.

Conclusions: The combined use of DIEP flap and GE-VLNT flaps in a single-stage procedure is a safe and reliable surgical option for patients with postmastectomy lymphedema who desire and are suitable for autologous microvascular breast and lymphatic reconstruction.

\footnotetext{
Keywords: Lymphedema; vascularized lymph node transfer (VLNT); breast reconstruction and lymph node transfer; deep inferior epigastric perforator flap and lymph node transfer (DIEP flap and lymph node transfer); gastroepiploic lymph node transfer
}

Submitted Nov 05, 2019. Accepted for publication Dec 12, 2019.

doi: $10.21037 /$ gs.2020.01.14

View this article at: http://dx.doi.org/10.21037/gs.2020.01.14 


\section{Introduction}

Upper extremity lymphedema (UEL) is a complex condition commonly related to breast cancer and its associated oncologic therapies, including cancer resection, radiation and chemotherapy. In the United States, breast cancer is the most common cancer diagnosis in women excluding cutaneous malignancies. Approximately 268,600 women are expected to be newly diagnosed with invasive breast cancer in 2019, and about 41,760 women in the United States are estimated to die from this cancer in the same year (1). Reported incidence of UEL among observational studies varies widely from $7.6 \%$ to $49 \%$ but is trending down due to less invasive cancer treatment options (2).

The mainstay of treatment for extremity lymphedema is complex decongestive therapy (CDT), which consists of an intensive program that combines bandaging, compression garments, exercise, manual lymphatic drainage, and selfcare measurements. If this fails, physiological or debulking surgical procedures are considered. The former attempts to restore or improve the lymphatic flow with procedures such as lymphatico-venous anastomoses (LVA) and vascularized lymph node transfer (VLNT) (3-9). On the other hand, the latter removes the diseased tissue through radical excision or suction-assisted lipectomy (SAL) (3). Both non-surgical and surgical treatment options aim to reduce the circumference of the affected extremity in addition to improve the patient's symptomology, extremity-related morbidities such as skin infections, and quality of life (10).

VLNTs have shown to be successful in treating patients with moderate to advanced stages of lymphedema $(6,7,9$, 11-16). Two possible theories have been described explaining its mechanism: the "lymphatic wick", which suggests the regeneration of spontaneous connections with efferent lymphatic, and the "lymphatic pump", which proposes an intrinsic lympho-venous shunt within the transferred lymph nodes (17).

Generally, breast reconstruction options encompass the use of tissue expanders/implants or autologous reconstruction based on abdominal, back, gluteal, or thigh flaps. For autologous breast reconstruction, the free deep inferior epigastric perforator (DIEP) flap has increasingly gained popularity due to its versatility, ample volume and comparative advantages over other flaps (18). A large study using the Mastectomy Reconstruction Outcomes Consortium (MROC) showed patient satisfaction with breasts and psychosocial and sexual well-being two years after surgeries were higher with autologous breast reconstruction (19). However, the odds of complications appear to be higher in this patient group (20).

Autologous breast reconstruction can be simultaneously performed with VLNTs in selected patients, requiring both breast reconstruction and surgical treatment for lymphedema (21-27). The most common vascularized lymph node flap used for this combined MBR and lymphatic reconstruction approach is the groin. In this study, we describe a novel combined surgical approach of autologous breast reconstruction with DIEP and gastroepiploic VLNT (GE-VLNT), and we present a case series of patients who underwent this procedure.

\section{Methods}

Between January 2018 and January 2019, all consecutive postmastectomy patients who underwent delayed autologous microvascular breast reconstruction (MBR) and were clinically and radiologically diagnosed with lymphedema, stage IIb-III International Society of Lymphology (ILS) (28) were included in this study. All patients underwent autologous breast reconstruction with the DIEP flap and gastroepiploic vascularized lymph node flap transfer by the lead author (PC) in the same operative time. Prior to surgery, the patients were examined by their oncologists, and all were considered cancer free. Patients older than 75 years, patients with prior surgical treatments for lymphedema or patients requiring bilateral breast reconstruction were excluded. Lymphedema was diagnosed preoperatively by thorough clinical evaluation, limb circumference measurements and lymphoscintigraphy, which served as a reference line to assess objectively response to surgery. Patient demographics, comorbidities, prior radiation therapy, operative characteristics, surgical outcomes and complications were prospectively collected and registered after obtaining approval from the local Ethics Committee.

Clinical photographs both pre- and post-operatively during follow-up were also documented. Circumferences of both the edematous extremity and the unaffected counterpart pre and at least 6 months postoperatively were documented to calculate the reduction rate. The anatomic landmarks used to measure the circumference were the following: $10 \mathrm{~cm}$ below the elbow or knee, $10 \mathrm{~cm}$ above the wrist or ankle, and at the midhand or midfoot. To calculate the circumference reduction rate, we used the mean of the circumferences measured. We defined the percentage of improvement as "reduction rate" comparing the affected 

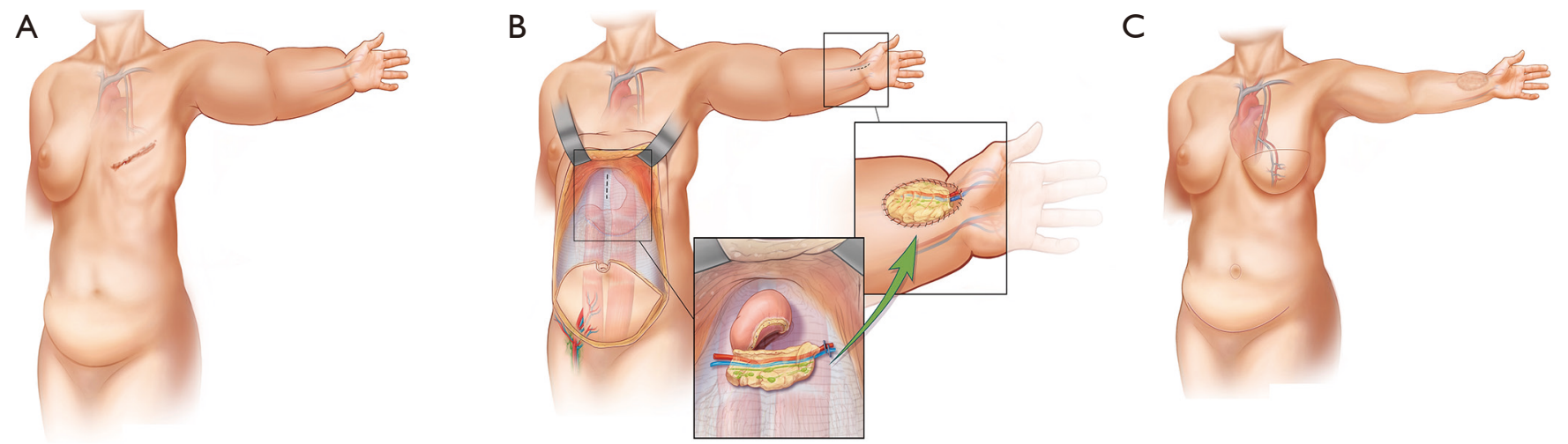

Figure 1 Schematic representation of the combined microvascular breast and lymphatic reconstruction. (A) Post-mastectomy female patient with left breast-cancer related lymphedema (BCRL). (B) The deep inferior epigastric perforator (DIEP) flap harvest. In the upper abdomen, a $6 \mathrm{~cm}$ incision is done over the linea alba to access the intra-abdominal space. Part of the stomach and omentum is exposed to harvest the gastroepiploic lymph node flap, which is then transferred to the wrist. (C) Immediate postoperative representation. Notice the reconstructed breast and the limb size reduction.

limb (AL) to the healthy limb (HL) using the following equation:

Circumferencereductionrate $(\%) \frac{[1-(\text { postperative } A L-H L)}{(\text { pre-postperative } A L-H L)]} \times 100$

\section{Surgical technique}

A two-surgical team approach was used. One team dissected and prepared the internal mammary vessels and the palmar branch of the radial artery and cephalic vein for its use as recipient vessels for the DIEP flap and the GE-VLNT, respectively, whereas the other team harvested the flaps. Figure 1 shows a schematic representation of the combined microvascular breast and lymphatic reconstruction.

Under general anesthesia, the DIEP flap, which we based on the deep inferior epigastric pedicle, was harvested in the standard fashion (29-31). Before dividing the deep inferior epigastric vascular pedicle, attention was then turned to the upper abdomen for the gastroepiploic vascularized lymph node flap harvest (Figure 2). The superior abdominal flap was dissected to the level of the xiphoid process. A midline laparotomy incision of about $6 \mathrm{~cm}$ was done along the linea alba. The greater curvature of the stomach and omentum were identified in the intraabdominal space and then extruded (Figure 2B). We cautiously injected $0.5 \mathrm{cc}$ of indocyanine green (ICG) into the omentum and identified the gastroepiploic lymph nodes. The dissection was carried along the greater curvature of the stomach from left to right to isolate the gastroepiploic vessels, and the flap was harvested, as previously described (13). The proximal portion of the lymph node flap based on the right gastroepiploic vessels were placed in the volar aspect of the wrist and anastomosed to the palmar branch of the radial artery and the cephalic vein. When needed, skin graft was used to cover the flap and close the skin without tension (Figure 2E). Extensive scar and fibrotic tissue were removed and released from the axillary vessels before DIEP flap inset. The DIEP flap was anastomosed to the internal mammary vessels. Adequate blood perfusion was evaluated clinically and with the use of Doppler for both flaps.

\section{Postoperative care}

All patients were admitted as inpatients for 5 to 7 days postoperatively for flap monitoring, pain control and physical therapy. Drains were removed when the output was less than $30 \mathrm{~mL} /$ day. Physiotherapy was started approximately 2 to 3 weeks postoperatively, after complete wound healing and suggested for 3 times a week for the next 6 months postoperatively.

\section{Statistical analysis}

Analyses were conducted using $\mathrm{JMP}^{\mathrm{O}}$ version 13 (SAS Institute Inc., Cary, NC, USA, 1989-2019). Measures of central tendency and dispersion were obtained to show data distribution. Wilcoxon signed-rank test was used to evaluate 

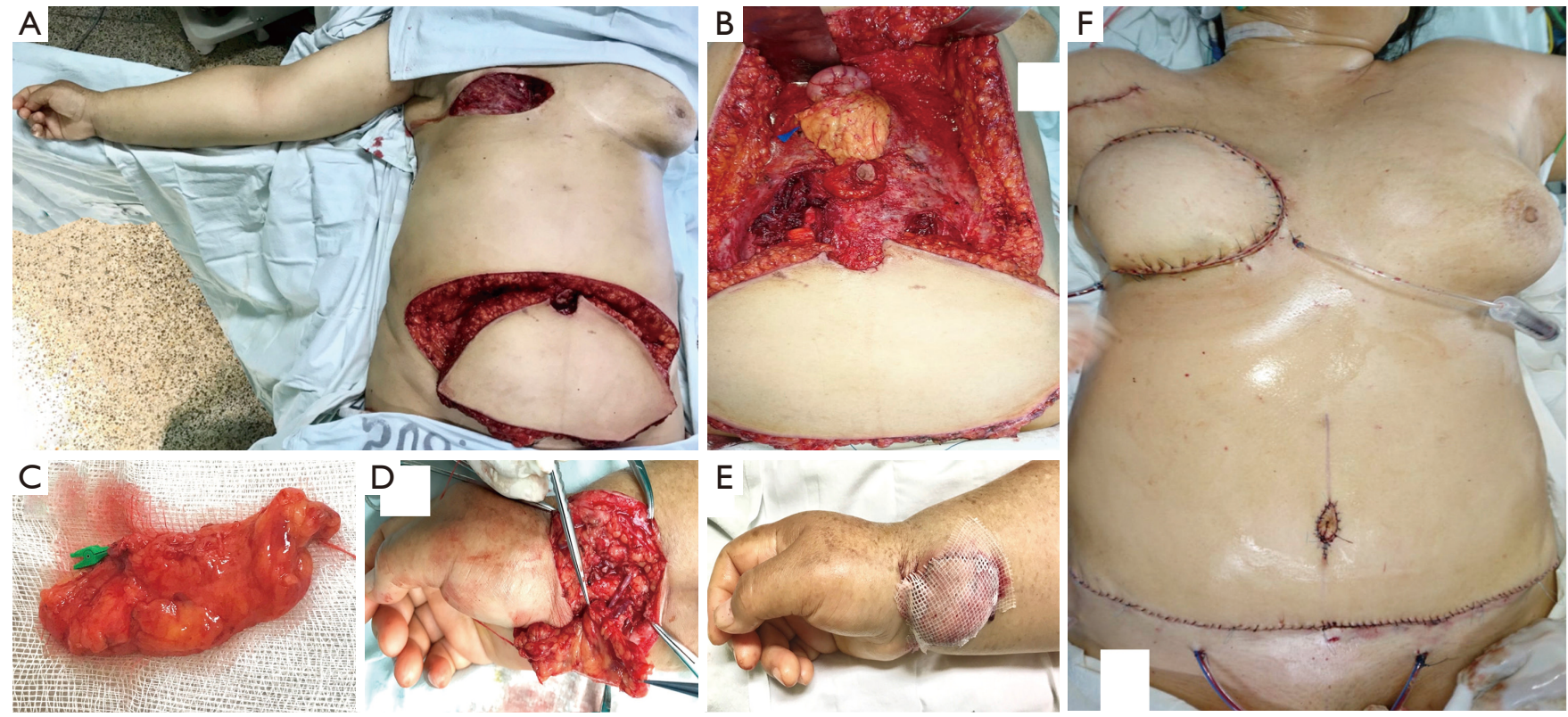

Figure 2 Intraoperative clinical pictures. (A) The deep inferior epigastric perforator (DIEP) flap harvest. (B) Mini-laparotomy performed after the elevation of the upper abdominal flap. Notice part of the stomach and omentum is extruded to harvest the gastroepiploic vascularized lymph node flap. (C) Transferred lymph node flap on the table. (D,E) Inset of the gastroepiploic lymph node flap at the level of the wrist and its closure with a split-thickness skin graft to avoid closure with tension. (F) Immediate postoperative picture after the combined microvascular breast reconstruction with DIEP flap and lymphatic reconstruction with GE-VLNT. GE-VLNT, gastroepiploic vascularized lymph node transfer.

pre and postoperative circumference measurements. Statistical significance level was set at $\mathrm{P}<0.05$.

\section{Results}

Six consecutive patients with refractory UEL related to breast cancer treatment underwent simultaneous MBR with DIEP flap and GE-VLNT. The mean age was $48 \pm$ 10.5 years and mean body mass index was $28.2 \pm 4.5 \mathrm{~kg} / \mathrm{m}^{2}$ (Table 1 ). Five patients $(83.3 \%)$ had received radiation therapy before surgery. The mean duration of lymphedema symptom was $18.6 \pm 5.3$ months. All patients underwent CDT for the treatment of extremity lymphedema with a mean duration of $15 \pm 1.3$ months before surgery.

For all patients, the internal mammary vessels were utilized as recipient vessels for the DIEP flap. In addition, the palmar branch of the radial artery and the cephalic vein at the level of the wrist were used as recipient vessels for the GE-VLNT in all patients. Patients were hospitalized for a mean duration of $15.8 \pm 2.5$ days. Mean follow-up time was $12.8 \pm 2.5$ months. Operative time is shown in Table 2 .

The mean circumference reduction rate was
$30.0 \% \pm 5.1 \%(\mathrm{P}<0.001)$. All patients required contralateral breast reduction for symmetry. One patient required re-exploration of the lymph node flap due to venous congestion secondary to kinking of pedicle vessels, but was successfully salvaged in the operating room redoing the anastomosis. There were no flap losses in our case series. No major complications were seen at the donor or recipient sites of the DIEP flap. One patient had small skin wound dehiscence in the abdominal donor site. All patients reported subjective symptomatic improvement. One patient with stage III lymphedema underwent additional liposuction of the affected extremity at 12 months postoperative to improve surgical outcomes (Table 3). Figures 3 and 4 show pre and postoperative pictures of two patients who underwent simultaneous autologous MBR and GE-VLNT.

\section{Discussion}

Surgical treatment for lymphedema has experienced a rapid growth over the past few years largely attributable to not only the increased awareness of this condition among patients and medical community, but also due to 
Table 1 Patient characteristics

\begin{tabular}{lc}
\hline Characteristic & Mean \pm SD/count \\
\hline Age, years & $48 \pm 10.5$ \\
Body mass index, $\mathrm{kg} / \mathrm{m}^{2}$ & $28.2 \pm 4.5$ \\
Duration of lymphedema-associated symptoms (months) & $18.6 \pm 5.3$ \\
Duration of preoperative complex decongestive therapy (months) & $15 \pm 1.3$ \\
Prior radiation therapy & $8.0-35.0$ \\
Yes & $12-17$ \\
No & 5 \\
\hline
\end{tabular}

$\mathrm{SD}$, standard deviation.

Table 2 Operative data

\begin{tabular}{lcc}
\hline Variable & Mean \pm SD/count & \multicolumn{1}{c}{ Range } \\
\hline Hospitalization (days) & $15.8 \pm 2.5$ & $12.5-18.0$ \\
Follow up time (months) & $12.8 \pm 2.5$ & $6.0-18.5$ \\
Operative time (min) & & \\
DIEP harvest & $105.5 \pm 15$ & $90-115$ \\
GE-VLN flap harvest & $35 \pm 5.6$ & $30.0-40.5$ \\
Total operative time & $325 \pm 25.5$ & $300.5-350.5$ \\
DIEP Flap weight (gram) & $380.4 \pm 62.3$ & $352-440$ \\
\hline
\end{tabular}

$\mathrm{SD}$, standard deviation; DIEP, deep inferior epigastric perforator; GE-VLN, gastroepiploic vascularized lymph nodes.

Table 3 Surgical outcomes and complications

\begin{tabular}{lcc}
\hline Variable & $\begin{array}{c}\text { Mean } \pm \\
\text { SD/count }\end{array}$ & Range \\
\hline Circumference reduction rate (\%) & $30.0 \pm 5.1$ & $18.0-39.7$ \\
Additional procedures after flap & & \\
transfer & 1 \\
Liposuction & 6 \\
Contralateral breast surgery for & \\
symmetry & \\
Complications & 0 \\
Seroma & 0 \\
Infection & 1 \\
Lymph node flap venous congestion & 1 \\
Abdominal donor-site wound & \\
dehiscence & \\
Flap failure & 0
\end{tabular}

SD, standard deviation. enhancements in microsurgical techniques. A simultaneous VLNT to treat lymphedema in patients who desire autologous MBR represents an enhancement in the comprehensive management of patients with breast cancerrelated lymphedema (BCRL). This approach enables the achievement of both objectives in a single-stage procedure: breast reconstruction and concomitant treatment of lymphedema. Previously published studies with the use of the groin lymph node flap have shown this simultaneous surgical approach is feasible, safe and have promising results in patients' quality of life and surgical outcomes $(21,22$, 24-27).

The first study describing the use of abdominalbased free tissue transfer for breast reconstruction and simultaneous vascularized lymph node flaps was published in 2012 by Saaristo et al. (26). In this study, both DIEP and muscle sparing transverse rectus abdominis musculocutaneous (msTRAM) flaps were harvested along with lymph nodes surrounding the superficial circumflex iliac vessels (SCIV). The abdominal flaps were anastomosed to the thoracodorsal vessels and the lymph node flaps were anastomosed to the retrograde thoracodorsal vessels if needed. Seven of their nine patients showed a significant reduction in circumferential extremity measurements and five out of six patients with radiologic evaluation showed improved flow in lymphoscintigraphy at 6 months postoperative compared with preoperative pictures.

Since the introduction of this combined surgical approach, diverse lymph node flaps within which the most common are the inguinal lymph nodes along the SCIV or superficial inferior epigastric vessels (SIEV) have been used. One of the most important comparative advantages offered by these lymph node flaps is that the DIEP flap can be raised with the groin flap as a chimeric flap and transferred en bloc 

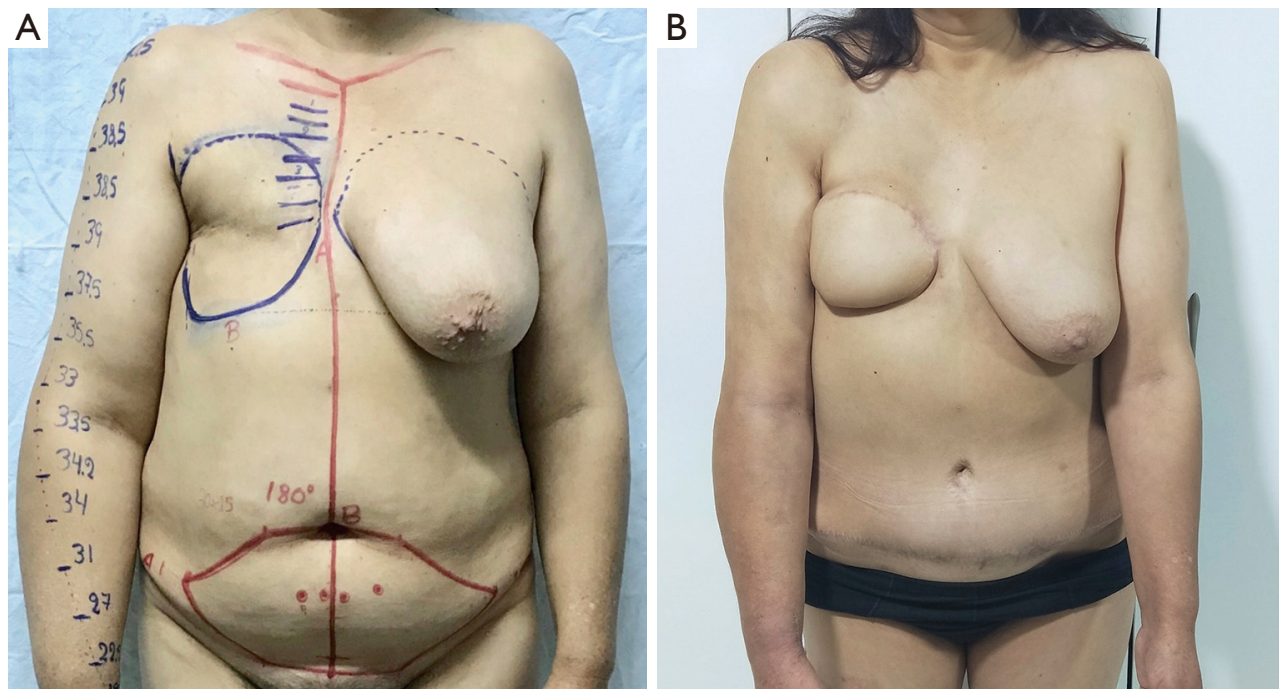

Figure 3 A 49-year-old female patient with left breast cancer-related lymphedema (BCRL) with 12 months of complex decongestive therapy (CDT) without significant improvement. The patient underwent the combined microvascular autologous breast reconstruction and gastroepiploic vascularized lymph node flap transfer. (A) Preoperative picture. Notice the mastectomy scar over her left chest and the volume and size discrepancy between the upper extremities. (B) Postoperative picture at 12-months follow-up evidencing a mean limb circumference reduction rate of $30 \%$. In addition, the patient reported improved lymphedema-associated symptoms.
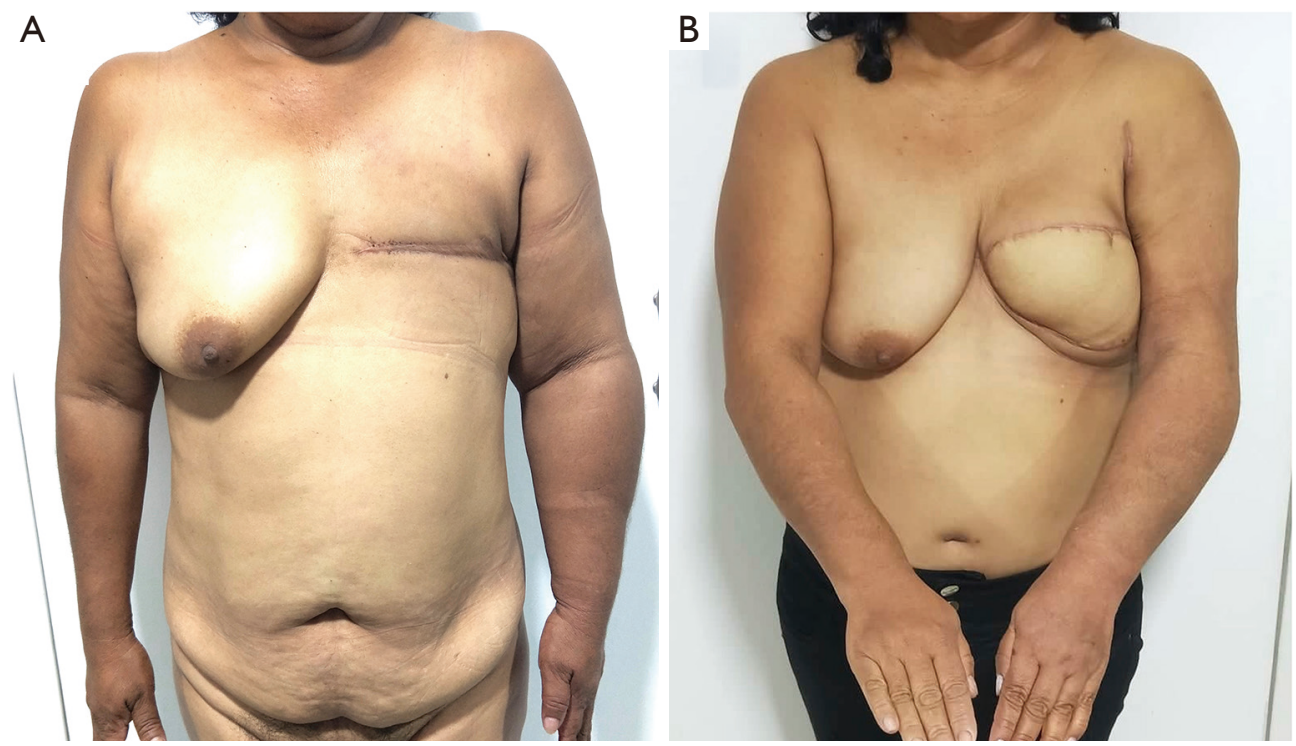

Figure 4 Pre and postoperative pictures of a 45 -year-old female patient with right breast cancer-related lymphedema (BCRL). She had received 15 months of complex decongestive therapy with unsatisfactory results. (A) Preoperative picture. (B) Postoperative picture at 12 months of follow-up showing a limb circumference reduction rate of $39 \%$.

to the recipient site. However, donor-site volume defect and risk of iatrogenic lymphedema are among its disadvantages. Careful dissection during flap harvest should be conducted to minimize the risk of iatrogenic lymphedema, which is one of the most dreaded complications of VLNT. In order to avoid harvest of sentinel lymph nodes draining the lower extremity and decrease the risk of iatrogenic lymphedema, reverse lymphatic mapping can guide the harvest of 
vascularized lymph node flaps. However, the risk of iatrogenic lymphedema is still latent (32). In addition, flaps based off of the SEIV have been reported to be associated with increased seroma formation $(26,33)$.

Due to the increased risk of iatrogenic lower extremity lymphedema and the restrictions on shaping of the reconstructed breast with the use of chimeric abdominal flaps, the use of GE-VLNT during autologous breast reconstruction emerges as a strong surgical alternative. In our study, we used this flap showing a significant mean circumference reduction rate $(30.0 \% \pm 5.1 \%, \mathrm{P}<0.001)$. To date, there have been no reports of iatrogenic lymphedema with the harvest of gastroepiploic vascularized lymph node flaps. It is versatile and contains a reliable amount of lymphatic tissue (at least three lymph nodes when $3 \mathrm{~cm}$ of surrounding tissue are obtained) (34). In addition, this flap can be divided into two isolated flaps based off of the two gastroepiploic vessels. These could be used as a doubleinset in patients with unilateral extremity lymphedema (23) or even in patients with bilateral UEL who desire autologous bilateral breast reconstruction. In addition, the bulky appearance of the flap at the recipient site can be improved with microsurgical debulking if necessary (4). In our case series, we used a single-level distal inset as these sites are usually healthy, have no scar tissue and are usually spared from the deleterious effects of radiation therapy. Additionally, axillary scar release was done as it has been shown to be an effective strategy in order to fasten the onset of improvement and positively impact patient's quality of life (35).

Even though the harvest of the gastroepiploic vascularized lymph node flap is relatively unchallenging and offers the above-mentioned versatility, this procedure is more invasive and entails a mini laparotomy of approximately $5-7 \mathrm{~cm}$. A detailed anatomical knowledge and a technically adequate abdominal wall closure are imperative to decrease donor site morbidity.

In our experience, for advanced lymphedema stages both the fluid and solid components of lymphedema should be addressed accordingly in order to obtain better outcomes. The VLNT adequately tackles fluid congestion and regenerates lymphatic connections; however, its role is still limited in cases where there is a high component of fibroadipose deposition. In these cases, a combined approach including SAL or another debulking procedure may be required. One of our patients underwent SAL procedure 12 months postoperatively to improved surgical outcomes.

This study adds to the increasing body of evidence supporting VLNT as an effective surgical treatment for lymphedema, particularly in patients undergoing breast reconstruction. However, it is not without limitations. The limited number of patients may not be representative and affects external validity. In addition, the follow-up time was relatively short. To the best of our knowledge, the present study is the largest case series with the longest follow-up combining microvascular breast and lymphatic reconstruction with DIEP flap and GE-VLNT.

\section{Conclusions}

The use of GE-VLNT for the treatment of lymphedema has shown promise in that there is no risk of iatrogenic lower extremity lymphedema. Based on our data, this is a safe and reliable procedure that adequately addresses two objectives: breast and lymphatic reconstruction. This combined surgical technique represents a great alternative to previously described donor-sites for the treatment of patients with BCRL who desire and are suitable for autologous MBR. This study paves the way for further prospective studies with longer follow-up to determine the true value of this combined surgical approach and to assess the differences between various donor flaps for VLNT in combination with MBR.

\section{Acknowledgments}

We thank the Division of Biomedical \& Scientific Visualization at the Mayo Clinic, Rochester, MN, for the design of the illustrations. This paper was partially presented at the 27th International Society of Lymphology Conference on September 23-26, 2019, in Buenos Aires, Argentina.

Funding: None.

\section{Footnote}

Provenance and Peer Review: This article was commissioned by the Guest Editors (Xiaona Lu, Antonio Jorge Forte) for the series "Lymphedema" published in Gland Surgery. The article was sent for external peer review organized by the Guest Editors and the editorial office.

Conflicts of Interest: All authors have completed the ICMJE uniform disclosure form (available at http://dx.doi. org/10.21037/gs.2020.01.14). The series "Lymphedema" was commissioned by the editorial office without any 
funding or sponsorship. AJF served as the unpaid Guest Editor of the series. The other authors have no other conflicts of interest to declare.

Ethical Statement: The authors are accountable for all aspects of the work in ensuring that questions related to the accuracy or integrity of any part of the work are appropriately investigated and resolved. The institutional review board approved the study (ALNH No: DMR005IRB-103) and written informed consent was obtained from all patients.

Open Access Statement: This is an Open Access article distributed in accordance with the Creative Commons Attribution-NonCommercial-NoDerivs 4.0 International License (CC BY-NC-ND 4.0), which permits the noncommercial replication and distribution of the article with the strict proviso that no changes or edits are made and the original work is properly cited (including links to both the formal publication through the relevant DOI and the license). See: https://creativecommons.org/licenses/by-nc-nd/4.0/.

\section{References}

1. Breastcancer.org. U.S. Breast Cancer Statistics. Understanding Breast Cancer 2019.

2. Meneses KD, McNees MP. Upper extremity lymphedema after treatment for breast cancer: a review of the literature. Ostomy Wound Manage 2007;53:16-29.

3. Ciudad P, Agko M, Huang TCT, et al. Comprehensive multimodal surgical treatment of end-stage lower extremity lymphedema with toe management: The combined Charles,' Homan's, and vascularized lymph node transfer (CHAHOVA) procedures. J Surg Oncol 2019;119:430-8.

4. Ciudad P, Sze-Wei Yeo M, Sapountzis S, et al. Microsurgical debulking procedure after free lymph node flap transfer. Microsurgery 2014;34:670-1.

5. Ciudad P, Manrique OJ, Date S, et al. Double gastroepiploic vascularized lymph node tranfers to middle and distal limb for the treatment of lymphedema. Microsurgery 2017;37:771-9.

6. Ciudad P, Manrique OJ, Date S, et al. A head-to-head comparison among donor site morbidity after vascularized lymph node transfer: Pearls and pitfalls of a 6-year single center experience. J Surg Oncol 2017;115:37-42.

7. Agko M, Ciudad P, Chen HC. Staged surgical treatment of extremity lymphedema with dual gastroepiploic vascularized lymph node transfers followed by suctionassisted lipectomy-A prospective study. J Surg Oncol 2018;117:1148-56.

8. Ciudad P, Agko M, Perez Coca JJ, et al. Comparison of long-term clinical outcomes among different vascularized lymph node transfers: 6-year experience of a single center's approach to the treatment of lymphedema. J Surg Oncol 2017;116:671-82.

9. Ciudad P, Manrique OJ, Bustos SS, et al. Comparisons in long-term clinical outcomes among patients with upper or lower extremity lymphedema treated with diverse vascularized lymph node transfer. Microsurgery 2020;40:130-6.

10. Garza R 3rd, Skoracki R, Hock K, et al. A comprehensive overview on the surgical management of secondary lymphedema of the upper and lower extremities related to prior oncologic therapies. BMC Cancer 2017; 17:468.

11. Ciudad P, Maruccia M, Socas J, et al. The laparoscopic right gastroepiploic lymph node flap transfer for upper and lower limb lymphedema: Technique and outcomes. Microsurgery 2017;37:197-205.

12. Ciudad P, Manrique OJ, Agko M, et al. Ileocecal vascularized lymph node transfer for the treatment of extremity lymphedema: A case report. Microsurgery 2019;39:81-4.

13. Ciudad P, Kiranantawat K, Sapountzis S, et al. Right gastroepiploic lymph node flap. Microsurgery 2015;35:496-7.

14. Yeo MS, Lim SY, Kiranantawat K, et al. A comparison of vascularized cervical lymph node transfer with and without modified Charles' procedure for the treatment of lower limb lymphedema. Plast Reconstr Surg 2014;134:171e-172e.

15. Sapountzis S, Singhal D, Rashid A, et al. Lymph node flap based on the right transverse cervical artery as a donor site for lymph node transfer. Ann Plast Surg 2014;73:398-401.

16. Ciudad P, Manrique OJ, Date S, et al. Double gastroepiploic vascularized lymph node tranfers to middle and distal limb for the treatment of lymphedema. Microsurgery 2017;37:771-9.

17. Tourani SS, Taylor GI, Ashton MW. Vascularized Lymph Node Transfer: A Review of the Current Evidence. Plast Reconstr Surg 2016;137:985-93.

18. Martinez CA, Reis SM, Rednam R, et al. The Outpatient DIEP: Safety and Viability following a Modified Recovery Protocol. Plast Reconstr Surg Glob Open 2018;6:e1898.

19. Santosa KB, Qi J, Kim HM, et al. Long-term PatientReported Outcomes in Postmastectomy Breast 
Reconstruction. JAMA Surg 2018;153:891-9.

20. Bennett KG, Qi J, Kim HM, et al. Comparison of 2-Year Complication Rates Among Common Techniques for Postmastectomy Breast Reconstruction. JAMA Surg 2018;153:901-8.

21. Nguyen AT, Chang EI, Suami H, et al. An Algorithmic Approach to Simultaneous Vascularized Lymph Node Transfer with Microvascular Breast Reconstruction. Ann Surg Oncol 2015;22:2919-24.

22. Duquette S, Socas J. Simultaneous Vascularized Lymph Node Transfer and Microsurgical Breast Reconstruction. In: Lymphedema: Surgical Approach and Specific Topics. Second Edition 2018:823.

23. Deldar R, Duquette S, Ceppa EP, et al. An alternative approach to combined autologous breast reconstruction with vascularized lymph node transfer. Microsurgery 2017;37:463-4.

24. Dancey A, Nassimizadeh A, Nassimizadeh M, et al. A chimeric vascularised groin lymph node flap and DIEP flap for the management of lymphoedema secondary to breast cancer. J Plast Reconstr Aesthet Surg 2013;66:735-7.

25. Chen R, Mu L, Zhang H, et al. Simultaneous breast reconstruction and treatment of breast cancer-related upper arm lymphedema with lymphatic lower abdominal flap. Ann Plast Surg 2014;73:S12-7.

26. Saaristo AM, Niemi TS, Viitanen TP, et al. Microvascular breast reconstruction and lymph node transfer for postmastectomy lymphedema patients. Ann Surg 2012;255:468-73.

27. De Brucker B, Zeltzer A, Seidenstuecker K, et al. Breast Cancer-Related Lymphedema: Quality of Life after Lymph

Cite this article as: Ciudad P, Manrique OJ, Bustos SS, Vargas MI, Reynaga C, Agko M, Huang TCT, Figueroa Benites E, Mayer HF, Forte AJ. Combined microvascular breast and lymphatic reconstruction with deep inferior epigastric perforator flap and gastroepiploic vascularized lymph node transfer for postmastectomy lymphedema patients. Gland Surg 2020;9(2):512-520. doi: 10.21037/gs.2020.01.14
Node Transfer. Plast Reconstr Surg 2016;137:1673-80.

28. Society TI, Document C, Congress XVII, et al. The diagnosis and treatment of peripheral lymphedema: 2016 consensus document of the International Society of Lymphology. Acta Angiol 2017;49:170-84.

29. Allen RJ, Treece P. Deep inferior epigastric perforator flap for breast reconstruction. Ann Plast Surg 1994;32:32-8.

30. Chevray PM. Update on Breast Reconstruction Using Free TRAM, DIEP, and SIEA Flaps. Semin Plast Surg 2004;18:97-104.

31. Nahabedian MY, Momen B, Galdino G, et al. Breast Reconstruction with the free TRAM or DIEP flap: patient selection, choice of flap, and outcome. Plast Reconstr Surg 2002;110:466-75.

32. Dayan JH, Dayan E, Smith ML. Reverse lymphatic mapping: a new technique for maximizing safety in vascularized lymph node transfer. Plast Reconstr Surg 2015;135:277-85.

33. Moradi P, Durrant C, Glass GE, et al. SIEA flap leads to an increase in abdominal seroma rates compared to DIEP flap for breast reconstruction. Eur J Plast Surg 2011;34:87-91.

34. Howell AC, Gould DJ, Mayfield C, et al. Anatomical Basis of the Gastroepiploic Vascularized Lymph Node Transfer: A Radiographic Evaluation Using Computed Tomographic Angiography. Plast Reconstr Surg 2018;142:1046-52.

35. Maruccia M, Elia R, Ciudad P, et al. Postmastectomy upper limb lymphedema: Combined vascularized lymph node transfer and scar release with fat graft expedites surgical and patients' related outcomes. A retrospective comparative study. J Plast Reconstr Aesthet Surg 2019;72:892-901. 